\title{
Emergent Gravity, Violated Relativity and Dark Matter
}

\author{
Yury F. Pirogov \\ Theory Division, Institute for High Energy Physics, Protvino, Russia \\ Email: yury.pirogov@ihep.ru \\ Received 7 May 2014; revised 2 June 2014; accepted 25 June 2014 \\ Copyright (C 2014 by author and Scientific Research Publishing Inc. \\ This work is licensed under the Creative Commons Attribution International License (CC BY). \\ http://creativecommons.org/licenses/by/4.0/ \\ c) (i) Open Access
}

\begin{abstract}
The nonlinear affine Goldstone model of the emergent gravity, built on the nonlinearly realized/ hidden affine symmetry, is concisely revisited. Beyond General Relativity, the explicit violation of general invariance/relativity, under preserving general covariance, is exposed. Dependent on a nondynamical affine connection, a generally covariant second-order effective Lagrangian for metric gravity is worked out, with the general relativity violation and the gravitational dark matter serving as the signatures of emergence.
\end{abstract}

\section{Keywords}

Spontaneous Symmetry Breaking, Nonlinear Realizations, Emergent Gravity, General Relativity Violation, Dark Matter

\section{Introduction}

It is widely accepted nowadays that General Relativity (GR) may be just (a piece of) an effective field theory of gravity to be ultimately superseded at the high (conceivably, as high as the Planck scale) energies by a more fundamental/underlying theory. At that, the conventional metric gravity could cease to be a priori existent, but, instead, would become an emergent/induced phenomenon. A lot of the drastically different approaches towards the emergence of gravity and space-time is presently conceivable ${ }^{1}$. In this paper, we work out an approach to the goal treating gravity as an affine Goldstone phenomenon in the effective field theory framework.

Typically, as a herald of an unknown high-energy theory there serves at the lower energies a nonlinear model. Being based on a nonlinearly realized/hidden symmetry, remaining linear on an unbroken subgroup, such a model could encounter in a concise manner for the spontaneously/dynamically broken symmetries of the fundamental theory. Inevitably, this occurs at the cost of more uncertainty and a partial loss of content. For the

\footnotetext{
${ }^{1}$ For recent surveys of the emergent gravity and space-time, see, e.g. [1] [2].
} 
global continuous internal symmetries, the nonlinear model framework was developed in [3] [4]. This approach proved to be extremely useful for studying, e.g., the so-called chiral model and played an important role in the advent of QCD as the true fundamental theory of strong interactions.

One might thus naturally expect that in the quest for an underlying theory of gravity GR should first be substituted by a nonlinear model. As such a model for gravity, aimed principally at reconstructing GR, there was originally proposed the model based on the nonlinearly realized/hidden affine symmetry, remaining linear on the unbroken Poincare subgroup [5] [6] $]^{2}$. In the context of emergence of gravity and space-time, the model was elaborated in [7] [8]. At that, reproducing GR the model may well include the GR violation [9] ${ }^{3}$. To this end, one should envisage in a field theory two kinds of fields - the dynamical/relative and nondynamical/absolute ones - and, respectively, two kinds of the diffeomorphism symmetries. First, the kinematical symmetry - the covariance restricts the mathematical form of the theory. Second, the dynamical symmetry - the general invariance/relativity serves as a gauge symmetry for gravity determining the physical content of the latter $[10]^{4}$. In $\mathrm{GR}$, without nondynamical fields, these notions coincide, both being the general ones. But beyond GR, in the presence of nondynamical fields, the notions differ. For consistency, the general covariance should better be preserved. On the other hand, the general invariance/relativity violation may well take place, serving as a source of the gravitational dark matter (DM). In the simplest case, such a scenario was worked out for the well-posed theory of gravity minimally violating GR to the unimodular relativity, with the scalar-graviton DM [9] [10]. Extending this scenario to other types of the GR violation and gravitational DM would thus be urgent.

In this paper, the model of emergent gravity based on the nonlinearly realized/hidden affine symmetry - the nonlinear affine Goldstone model - is systematically revisited. To allow for the GR violation, two kinds of coordinates - the absolute/background and relative/observer's ones—are envisaged. A generally covariant secondorder effective Lagrangian for the emergent metric gravity, dependent on a nondynamical affine connection, is consistently worked out in the most general fashion, with a limited version discussed in more detail. The model is proposed as a prototype for the emergent gravity and space-time, with the GR violation and the gravitational DM serving as the signatures of emergence. In Section 2, the nonlinear realizations are applied in the context of emergent gravity on an arbitrary nondynamical affine background. In Section 3, an effective Lagrangian of emergent metric gravity is worked out and discussed. Appendix A shortly describes the general techniques of nonlinear realizations for the spontaneous/dynamical symmetry breaking. Appendix B presents a short compendium of conventions and notations.

\section{Nonlinear Realizations and Emergent Gravity}

\subsection{Gravity as an Affine Goldstone Phenomenon}

In the case at hand in the paper, an underlying theory of gravity is to be originally invariant under the global affine symmetry group $G=I G L(d, R), d=4^{5}$. Eventually, the symmetry spontaneously/dynamically brakes down to the Poincare one, assumed to be exact:

$$
G=I G L(d, R) \rightarrow H=I S O(1, d-1) .
$$

A putative mechanism of such a breaking is beyond the scope of the nonlinear model. According to general theory (see, Appendix A), the breaking results in the nonlinear realization of the affine symmetry on the coset space $G / H=I G L(d, R) / I S O(1, d-1)$, with the $d(d+1) / 2$-component coset elements. The Goldstone boson of the respective nonlinear realization is to be treated as a primary gravity field. To consistently apply the nonlinear realization technique to such a global external symmetry, a two-stage procedure is to be implemented, starting from a flat affine background and extending then onto a curved one.

\subsection{Flat Affine Background}

First, let $\mathcal{R}_{d} \simeq R^{d}$ be a $d$-dimensional homogeneous space, with the affine group of motions, $\mathcal{R}_{d} \rightarrow R_{d}$. By

\footnotetext{
${ }^{2}$ For a fiber bundle formalism, cf. [7].

${ }^{3}$ To characterize such a phenomenon, the term "general covariance violation" used in [9] is to be more appropriately substitutes by the "general invariance/relativity violation" [10] used in what follows.

${ }^{4}$ For a discussion of the general covariance vs. general invariance, cf. also [11].

${ }^{5}$ The following consideration is formally independent of $d \geq 2$.
} 
default, $\mathcal{R}_{d}$ admits the globally affine coordinates $\xi^{m} \in R^{d}, m=0,1, \cdots, n-1^{6}$, undergoing the affine transformations:

$$
\xi^{m} \stackrel{(A, a)}{\mathrm{TM}} \xi^{\prime m}=\xi^{n} A_{n}^{-1 m}+a^{m},
$$

with the arbitrary constant parameters $A_{n}^{m}$ and $a^{m}$ for the (reversible) linear deformations and translations, respectively ${ }^{7}$. This space will serve as the representation one for constructing the nonlinear model. In accord with the general formalism, there are two modes for realization of the hidden affine symmetry: the nonlinear and linearized ones.

\subsubsection{Pseudo-Symmetric Nonlinear Realization}

A coset element $\vartheta_{m}^{a}, a=0,1, \cdots, d-1$, may uniquely be chosen to be pseudo-symmetric, i.e.,

$$
\eta^{a m} \vartheta_{m}^{b}=\eta^{b m} \vartheta_{m}^{a}
$$

(at least in a suitable neighborhood of $\vartheta_{m}^{a}=\delta_{m}^{a}$, where this condition is evidently fulfilled). Here, $\eta^{a b}$ (and $\left.\eta_{a b}\right)$ is the invariant under $S O(1, d-1)$ Minkowski symbol, by which the globally Lorentzian indices $a, b$, etc., are manipulated. Under $A \in G L(d, R)$ the coset element should transform nonlinearly as

$$
\vartheta_{m}^{a}(\xi) \stackrel{A}{\longrightarrow} \vartheta_{m}^{\prime a}\left(\xi^{\prime}\right)=A_{m}^{n} \vartheta_{n}^{b}(\xi) \Lambda_{b}^{\prime-1 a}(A, \vartheta),
$$

with $\Lambda^{\prime} \in S O(1, d-1) \subset G L(d, R)$ chosen so to retain the pseudo-symmetry after action of $A$. At that, due to $\Lambda_{a b}=\Lambda_{b a}^{-1}$ there automatically fulfills the linearity condition $\Lambda^{\prime}(\Lambda, \vartheta)=\Lambda$ for any $\Lambda \in S O(1, d-1)^{8}$. Present the symmetric Lorentz tensor $\vartheta^{a b} \equiv \eta^{a m} \vartheta_{m}^{b}$ in terms of a symmetric tensor $h^{a b}$ as $\vartheta \equiv \exp (h / 2)$, where, e.g., $(h h)^{a b}=h^{a c} h^{b d} \eta_{c d}$, etc. With $H=S O(1, d-1)$ serving as a classification group, one can treat $h^{a b}$, with $d(d+1) / 2$ independent components, as a tensor Goldstone boson emerging under breaking the global affine symmetry to the Poincare one.

\subsubsection{Locally Lorentzian Linear Representation}

The affine Goldstone model gets simplified with the nonlinear realization being linearized in terms of a $d^{2}$-component frame-like field $\vartheta_{m}^{\alpha}, \alpha=0,1, \cdots, n-1$ (and its inverse $\vartheta_{\alpha}^{m}$ ). The field transforms under $A \in G L(d, R)$ as

$$
\vartheta_{m}^{\alpha}(\xi) \stackrel{A}{\longrightarrow} \vartheta_{m}^{\prime \alpha}\left(\xi^{\prime}\right)=A_{m}^{n} \vartheta_{n}^{\beta}(\xi) \Lambda_{\beta}^{-1 \alpha}(\xi)
$$

(and likewise for $\vartheta_{m}^{\alpha}$ ), modulo an arbitrary $\Lambda_{\alpha}^{\beta}(\xi) \in S O(1, d-1)_{\text {loc }}$ satisfying $\Lambda_{\alpha \beta}=\Lambda_{\beta \alpha}^{-1}$, with the invariant $\eta_{\alpha \beta}$. Due to invariance under $S O(1, d-1)_{\text {loc }}$, with the $d(d-1) / 2^{\text {loc }}$ local parameters, the number of the independent components remains in fact the same $d(d+1) / 2$. Explicitly, one can impose a Lorentz gauge by projecting $\vartheta_{m}^{\alpha} \rightarrow \vartheta_{m}^{a}=\vartheta_{m}^{\beta} \Lambda_{\beta}^{-1 a}(\vartheta)$ so that $\vartheta_{m}^{\alpha}$ becomes pseudo-symmetric. Thus, the two realization modes, the nonlinear and linearized ones, are equivalent. However, the linear representation may be more advantageous due to grasping all the equivalent nonlinear realizations.

Restricting ourselves to the pure gravity, introduce the generic action element produced by an infinitesimal neighborhood $d^{d} \xi$ of a reference point $\Xi$ as follows:

$$
\mathrm{d} S=\mathcal{L}_{g} d^{d} \xi \equiv L_{g}\left(\vartheta_{m}^{\alpha}, \partial_{n} \vartheta_{m}^{\alpha}, \cdots\right)\left|\operatorname{det}\left(\vartheta_{m}^{\alpha}\right)\right| \mathrm{d}^{d} \xi,
$$

with $\mathrm{d} S$ being invariant relative to $G L(d, R) \otimes S O(1, n-1)_{\text {loc }}$. For the pure gravity, the field $\vartheta_{m}^{\alpha}$ may be converted into the symmetric Lorentz-invariant second-rank affine tensor $g_{m n}$ with $d(d+1) / 2$ independent degrees of freedom:

$$
g_{m n}(\xi)=\vartheta_{m}^{\alpha} \eta_{\alpha \beta} \vartheta_{n}^{\beta} .
$$

In particular, one has $\left|\operatorname{det}\left(\vartheta_{m}^{\alpha}\right)\right|=\sqrt{\left|\operatorname{det}\left(g_{m n}\right)\right|}$. This tensor may eventually be treated as the emergent metric

\footnotetext{
${ }^{6}$ Being here just a notation, the index $m=0$ is understood to subsequently compile with the unbroken Lorentz subgroup.

${ }^{7}$ Remaining unbroken, the translation part of the symmetry is omitted in what follows.

${ }^{8}$ Moreover, the dilatations $R$ are represented linearly, too, with the trivial compensating factor, $\Lambda^{\prime}(R, \vartheta)=I$.
} 
being absent prior to the affine symmetry breaking. Transforming the result to the arbitrary curvilinear coordinates $x^{\mu}=x^{\mu}(\xi)$ on $\mathcal{R}_{d}$ and integrating over $\mathcal{R}_{d}$ one would get the nonlinear model of gravity on the flat affine background. To account for a more general topology, one should go over to a curved affine background, with the action element given by Equation (6) used as a local flat counterpart.

\subsection{Curved Affine Background}

Let now $M_{d}$ be a $d$-dimensional differentiable "world" manifold marked with some "observer's" coordinates $x^{\mu} \in R^{d}, \quad \mu=0,1, \cdots, n-1^{9}$. Let $\mathcal{M}_{d}$ be moreover endowed with a nondynamical affine connection $\hat{\Gamma}_{\mu \nu}^{\lambda}(x)$, free of torsion, $\hat{\Gamma}_{\mu \nu}^{\lambda}-\hat{\Gamma}_{v \mu}^{\lambda}=0$. Such a background affine "texture" is to be formed by an underlying theory on par with the affine symmetry breaking. In a vicinity of a fixed, but otherwise arbitrary point $X$ the connection may be decomposed as follows

$$
\hat{\Gamma}_{\mu \nu}^{\lambda}(x)=\hat{\Gamma}_{\mu \nu}^{\lambda}(X)+\frac{1}{2} \hat{R}_{\mu \rho \nu}^{\lambda}(X)(x-X)^{\rho}+\mathcal{O}\left((x-X)^{2}\right),
$$

where $\hat{R}_{\mu \rho \nu}^{\lambda}(X)$ is the background curvature tensor in the reference point $X$. In a patch around $X$, choose on $\mathcal{M}_{d}$ some coordinates $\xi_{X}^{m}=\xi_{X}^{m}(x)$ (having the inverse $x^{\mu}=x^{\mu}\left(\xi_{X}\right)$ ) so that conventionally

$$
\hat{\Gamma}_{m n}^{l}\left(\xi_{X}\right)=e_{m}^{\mu} e_{n}^{v} e_{\lambda}^{l}(x)\left(\hat{\Gamma}_{\mu v}^{\lambda}(x)-e_{r}^{\lambda}(x) \partial^{2} \xi_{X}^{r} / \partial x^{\mu} \partial x^{v}\right),
$$

where $e_{\mu}^{m}(x)=\partial \xi_{X}^{m} / \partial x^{\mu}$ and $e_{m}^{\mu}(x)=\partial x^{\mu} /\left.\partial \xi_{X}^{m}\right|_{\xi_{X}=\xi_{X}(x)}$. Differentiating the reversibility relations $\xi_{X}^{m}\left(x\left(\xi_{X}\right)\right)=\xi_{X}^{m}$ and $x^{\mu}\left(\xi_{X}(x)\right)=x^{\mu}$ one gets $e_{\lambda}^{m} e_{n}^{\lambda}=\delta_{n}^{m}$ and $e_{l}^{\mu} e_{v}^{l}=\delta_{v}^{\mu}$. More particularly, adjust the coordinates $\xi_{X}^{m}$ as follows:

$$
\xi_{X}^{l}=\Xi_{X}^{l}+e_{\lambda}^{l}(X)\left((x-X)^{\lambda}+\frac{1}{2} \hat{\Gamma}_{\mu \nu}^{\lambda}(X)(x-X)^{\mu}(x-X)^{v}\right)+\mathcal{O}\left((x-X)^{3}\right),
$$

implying

$$
e_{l}^{\lambda}(X) \partial^{2} \xi_{X}^{l} /\left.\partial x^{\mu} \partial x^{\nu}\right|_{x=X}=\hat{\Gamma}_{\mu \nu}^{\lambda}(X) .
$$

In view of Equation (9) one thus gets $\hat{\Gamma}_{m n}^{l}\left(\Xi_{X}\right)=0$. Moreover, in view of Equation (8) in coordinates $\xi_{X}^{m}$ one has

$$
\hat{\Gamma}_{m n}^{l}\left(\xi_{X}\right)=\frac{1}{2} \hat{R}_{m r n}^{l}\left(\Xi_{X}\right)\left(\xi_{X}-\Xi_{X}\right)^{r}+\mathcal{O}\left(\left(\xi_{X}-\Xi_{X}\right)^{2}\right) .
$$

The manifold $\mathcal{M}_{d}$ looking in the coordinates $\xi_{X}^{m}$ approximately flat around $\Xi_{X}$, call such coordinates the locally affine ones attached to the point $X$, with $e_{\mu}^{m}$ and $e_{m}^{\mu}$ being the respective locally affine frames. In these coordinates, project a patch of $\mathcal{M}_{d}$ around $X$ onto the representation space $\mathcal{R}_{d}$ (associating the latter with the tangent space $\mathcal{T}_{X d}$ in $\left.X\right)$ through $\xi^{m}-\Xi^{m}=\xi_{X}^{m}-\Xi_{X}^{m}+\mathcal{O}\left(\left(\xi_{X}-\Xi_{X}\right)^{2}\right)$. This maps in the leading approximation the action element given by Equation (6) from $\mathcal{R}_{d}$ onto $\mathcal{M}_{d}$. Transform then the local result around $\Xi_{X}$ from coordinates $\xi_{X}^{m}$ to the arbitrary observer's coordinates $x^{\mu}$ by means of substitutions $\vartheta_{m}^{\alpha}=e_{m}^{\mu} \vartheta_{\mu}^{\alpha}, \partial_{m}=e_{m}^{\mu} \partial_{\mu}$ and $\mathrm{d}^{d} \xi_{X}=\left|\operatorname{det}\left(e_{\mu}^{m}\right)\right| \mathrm{d}^{d} x$, with frames $\epsilon_{m}^{\mu}(x)$ and $\epsilon_{\mu}^{m}(x)$. Integrating finally over $\mathcal{M}_{d}$ one gets the generic gravity action (redefining $X \rightarrow x$ ) as follows:

$$
S=\int L_{g}\left(\vartheta_{\mu}^{\alpha}, \partial_{\nu} \vartheta_{\mu}^{\alpha}, \cdots ; \hat{\Gamma}_{\mu \nu}^{\lambda}\right)\left|\operatorname{det}\left(\vartheta_{\mu}^{\alpha}\right)\right| \mathrm{d}^{d} x \ldots
$$

Evidently, the locally Lorentzian frame $\vartheta_{\mu}^{\alpha}$ satisfies the reversibility relations $\vartheta_{\mu}^{\alpha} \vartheta_{\beta}^{\mu}=\delta_{\beta}^{\alpha}$ and $\vartheta_{\alpha}^{\mu} \vartheta_{v}^{\alpha}=\delta_{v}^{\mu}$. Due to $\vartheta_{\mu}^{\alpha}=e_{\mu}^{m} \vartheta_{m}^{\alpha}$ the frame transforms under the diffeomorphisms $x^{\mu} \rightarrow x^{\prime \mu}=x^{\prime \mu}(x)$ modulo an arbitrary

${ }^{9}$ The index $\mu=0$ here is just a notation acquiring the physical meaning after the emergence of metric. 
$\Lambda \in S O(1, d-1)_{\mathrm{loc}}$ as

$$
\vartheta_{\mu}^{\alpha}(x) \rightarrow \vartheta_{\mu}^{\prime \alpha}\left(x^{\prime}\right)=\frac{\partial x^{\nu}}{\partial x^{\prime \mu}} \vartheta_{v}^{\beta}(x) \Lambda_{\beta}^{-1 \alpha}(x), .
$$

(and likewise for $\vartheta_{\alpha}^{\mu}=e_{m}^{\mu} \vartheta_{\alpha}^{m}$ ). The action given by Equation (13), as originated from Equation (6), is to be invariant relative to the local Lorentz transformations, being at the same time generally covariant. At that, the locally Lorentzian frame $\vartheta_{\mu}^{\alpha}$ is to be treated as the dynamical one, while the background affine connection $\hat{\Gamma}_{\mu v}^{\lambda}$ as nondynamical. Otherwise, under extremizing $S$, the background connection should not be varied, $\delta \hat{\Gamma}_{\mu \nu}^{\lambda}=0$. Under the requirement of the background-independence, the action $S$ would preserve the general diffeomorphism invariance/relativity. In the case of the residual dependence on the background $\hat{\Gamma}_{\mu \nu}^{\lambda}$, the action, though retaining the general covariance, violates, partially or totally, the general invariance/relativity.

\section{Emergent Metric}

Restricting himself by the pure gravity one can choose as an independent variable for gravity, instead of the Lorentzian frame $\vartheta_{\mu}^{\alpha}$, its bilinear Lorentz-invariant combination

$$
g_{\mu v}(x)=\vartheta_{\mu}^{\alpha} \eta_{\alpha \beta} \vartheta_{v}^{\beta}=e_{\mu}^{m} \vartheta_{m}^{\alpha} \eta_{\alpha \beta} \vartheta_{n}^{\beta} e_{v}^{n}=e_{\mu}^{m} g_{m n} e_{v}^{n}, .
$$

with $\left|\operatorname{det}\left(\vartheta_{\mu}^{\alpha}\right)\right|=\sqrt{\left|\operatorname{det}\left(g_{\mu v}\right)\right|^{10}}$ At that, the frame $\vartheta_{\mu}^{\alpha}$, of which $g_{\mu v}$ is composed, though being the primary gravity field, manifests itself explicitly only in interactions with matter (omitted here). The tensor field $g_{\mu v}$ may be treated as an emergent metric. It is the emergence of metric which converts a background manifold $\mathcal{M}_{d}$ with an affine connection into the true space-time, the latter becoming in a sense emergent, as well ${ }^{11}$.

\section{Violated Relativity and Dark Matter}

\subsection{Affine Symmetry}

Building the real nonlinear model starts out from $\mathcal{R}_{d}$ in the globally affine coordinates $\xi^{m}$. To construct the Lagrangian dependent on the affine tensor $g_{m n}$ and its derivatives construct first the Christoffel-like affine tensor

$$
\Gamma_{m n}^{l}(\xi)=\frac{1}{2} g^{l k}\left(\partial_{m} g_{n k}+\partial_{n} g_{m k}-\partial_{k} g_{m n}\right) .
$$

A derivative of $g_{m n}$ may uniquely be expressed through a combination of $\Gamma_{m n}^{l}$ (and v.v.). By means of the latter, one can construct the Riemann tensor $R_{m r n}^{l}$, the Ricci tensor $R_{m n}=R_{m l n}^{l}$ and the Ricci scalar $R=g^{m n} R_{m n}$. Altogether, one can construct on $\mathcal{R}_{d}$ the generic affine-invariant second-order effective Lagrangian for gravity

$$
L_{g}=\frac{1}{2} \kappa_{g}^{2}\left(L_{0}+\sum_{i=1}^{5} \varepsilon_{i} \Delta L_{i}\right), .
$$

with the partial bilinear in $\Gamma_{m n}^{l}$ contributions as follows:

$$
\begin{aligned}
& L_{0}=2 \Lambda-R\left(g_{m n}, \Gamma_{m n}^{l}\right), \Delta L_{1}=g^{m n} \Gamma_{m k}^{k} \Gamma_{n l}^{l}, \\
& \Delta L_{2}=g_{m n} g^{k l} g^{r s} \Gamma_{k l}^{m} \Gamma_{r s}^{n}, \Delta L_{3}=g^{m n} \Gamma_{m n}^{k} \Gamma_{k l}^{l}, \quad \\
& \Delta L_{4}=g_{m n} g^{k l} g^{r s} \Gamma_{k r}^{m} \Gamma_{l s}^{n}, \Delta L_{5}=g^{m n} \Gamma_{m k}^{l} \Gamma_{n l}^{k} .
\end{aligned}
$$

For completeness, there is included into $L_{0}$ a constant $\Lambda$. The parameter $\kappa_{g}=1 / \sqrt{8 \pi G}$ is the Planck mass, with $G$ being the Newton's constant, and $\varepsilon_{i}, i=1, \cdots, 5$, are the dimensionless free parameters. The pre-

\footnotetext{
${ }^{10} \mathrm{By}$ this token, one can use the convectional relation $e_{\mu}^{m}=g^{m n} g_{\mu v} e_{n}^{v}$, etc.

${ }^{11}$ the same vein, one could formally consider other patterns of the affine symmetry breaking, $G L(d, R) \rightarrow S O(n, d-n), n=0,1, \cdots,[d / 2]$, resulting in the same number of the affine Goldstone bosons, with the temporal-spatial signature $(n, d-n)$ of the emergent metric to be eventually selected [8]. For $d=4$, cf., e.g. [12].
} 
sented terms exhaust all the bilinear in $\Gamma_{m n}^{l}$ second-order ones admitted by the affine symmetry, with no prior preference among them ${ }^{12}$. Transforming the results to the curvilinear coordinates $x^{\mu}$ on $\mathcal{R}_{d}$ one would arrive at a generally covariant theory of gravity on a flat affine background. However, this is no more than a limited version of a more general case (see, further on).

\subsection{General Covariance}

Let us then map the above results from $\mathcal{R}_{d}$ onto $\mathcal{M}_{d}$, first, in the locally affine coordinates $\xi_{X}^{m}$ around $\Xi_{X}$ through the identical substitution $\Gamma_{m n}^{l}(\xi) \rightarrow \Gamma_{m n}^{l}\left(\xi_{X}\right)$ and then in the arbitrary observer's coordinates $x^{\mu}$ around $X$ (using for $\Gamma_{m n}^{l}\left(\Xi_{X}\right)$ the counterpart of Equation (9) supplemented by Equation (11)). Altogether, we get the relation

$$
\Gamma_{m n}^{l}\left(\Xi_{X}\right)=e_{m}^{\mu} e_{n}^{v} e_{\lambda}^{l}(X)\left(\Gamma_{\mu \nu}^{\lambda}(X)-\hat{\Gamma}_{\mu \nu}^{\lambda}(X)\right),
$$

where conventionally (with $X \rightarrow x$ )

$$
\Gamma_{\mu \nu}^{\lambda}(x)=\frac{1}{2} g^{\lambda \rho}\left(\partial_{\mu} g_{v \rho}+\partial_{\nu} g_{\mu \rho}-\partial_{\rho} g_{\mu v}\right) \text {. }
$$

is the Christoffel connection corresponding to metric $g_{\mu \nu}(x)$. Altogether, the most general second-order generally covariant effective Lagrangian for the emergent metric gravity is given by Equation (17), with ${ }^{13}$

$$
\begin{aligned}
& L_{0}=2 \Lambda-R\left(g_{\mu \nu}, \Gamma_{\mu \nu}^{\lambda}\right), \Delta L_{1}=g^{\mu v} B_{\mu \kappa}^{\kappa} B_{v \lambda}^{\lambda}, \\
& \Delta L_{2}=g_{\mu \nu} g^{\kappa \lambda} g^{\rho \sigma} B_{\kappa \lambda}^{\mu} B_{\rho \sigma}^{v}, \Delta L_{3}=g^{\mu v} B_{\mu \nu}^{\kappa} B_{\kappa \lambda}^{\lambda}, \\
& \Delta L_{4}=g_{\mu \nu} g^{\kappa \lambda} g^{\rho \sigma} B_{\kappa \rho}^{\mu} B_{\lambda \sigma}^{v}, \Delta L_{5}=g^{\mu v} B_{\mu \kappa}^{\lambda} B_{v \lambda}^{\kappa},
\end{aligned}
$$

dependent on the generally covariant tensor

$$
B_{\mu v}^{\lambda}(x) \equiv \Gamma_{\mu \nu}^{\lambda}-\hat{\Gamma}_{\mu v}^{\lambda} .
$$

The background-independent term $L_{0}$ corresponds to GR with a cosmological constant $\Lambda$, while the background-dependent ones $\Delta L_{i}, i=1, \cdots, 5$, to the GR violation. The theory of gravity with the GR-violating effective Lagrangian given by Equations (17), (21) and (22) may be referred to as Violated Relativity (VR). Most generally, it depends on the $d^{2}(d+1) / 2$ nondynamical functions besides the five constant Lagrangian parameters $\varepsilon_{i}{ }^{14}$.

\subsection{Gravitational DM}

Varying the gravity action with respect to $g_{\mu v}$, under fixed $\hat{\Gamma}_{\mu \nu}^{\lambda}$, we get the vacuum gravity field equations in a generic form as follows:

$$
R_{\mu \nu}-\frac{1}{2} R g_{\mu \nu}+\Lambda g_{\mu v}=\frac{1}{\kappa_{g}^{2}} \sum_{i}^{5} \varepsilon_{i} \Delta T_{\mu \nu}^{(i)}\left(g_{\rho \sigma}, B_{\rho \sigma}^{\lambda}\right) \equiv \frac{1}{\kappa_{g}^{2}} \Delta T_{\mu \nu}
$$

Here $\Delta T_{\mu \nu}^{(i)}$ are the generally covariant contributions to the equations due to $\Delta L_{i}$ :

$$
\Delta T^{(i) \mu v}=\frac{2}{\sqrt{|g|}} \frac{\delta\left(\sqrt{|g|} \Delta L_{i}\right)}{\delta g_{\mu \nu}},
$$

\footnotetext{
${ }^{12}$ On the affine symmetry reason, one could add two more linear terms, $g^{m n} \partial_{l} \Gamma_{m n}^{l}$ and $g^{m n} \partial_{m} \Gamma_{n l}^{l}$, which however may be expressed though the rest of the terms modulo surface contributions.

${ }^{13}$ The terms without derivatives of $g_{\mu v}$, as well as the extra factors given by the powers of $g=\operatorname{det}\left(g_{\mu v}\right)$, are forbidden by the hidden affine symmetry.

${ }^{14}$ Treating $\Delta L_{i}$ as the small perturbations and $L_{0}$ as the leading term, one can extend the latter by the higher-order generally covariant contributions.
} 
with $g=\operatorname{det}\left(g_{\mu v}\right)$ and $\delta / \delta g_{\mu v}$ designating a total variational derivative. The r.h.s. of Equation (23) may formally be treated as a covariantly conserved, $\nabla_{\mu} \Delta T^{\mu \nu}=0$, canonical energy-momentum tensor of the gravitational DM due to the GR violation $\left(\varepsilon_{i} \neq 0\right)$. An affine texture of space-time due to $\hat{\Gamma}_{\mu \nu}^{\lambda}$, resulting in such a phenomenon, signifies ultimately the gravity and space-time as emergent.

\subsection{Limited GR Violation}

Generally, the phenomenological study of VR is rather cumbersome. To simplify it as much as possible, consider the formal limit $\hat{\Gamma}_{\mu v}^{\lambda}=0$ corresponding to the flat affine background in the globally affine coordinates. The various observations being in reality fulfilled in the different coordinates, it is practically impossible for an observer to guess/use the unknown globally affine coordinates $a b$ initio. In the lack of this knowledge, one should start from suitable observer's coordinates $x^{\mu}$, assuming some $\hat{\Gamma}_{\mu \nu}^{\lambda}(x)$, to eventually reveal, with the help of observations, the globally affine coordinates $\xi^{m}$ (independent of any reference point $X$ ), so that $\hat{\Gamma}_{m n}^{l}(\xi) \equiv 0$. According to Equation (9), one should have in this case the restriction

$$
\hat{\Gamma}_{\mu \nu}^{\lambda}(x)=\left.\frac{\partial^{2} \xi^{l}}{\partial x^{\mu} \partial x^{v}} \frac{\partial x^{\lambda}}{\partial \xi^{l}}\right|_{\xi=\xi(x)}=e_{l}^{\lambda} \partial_{\mu} e_{\nu}^{l},
$$

Here $e_{\mu}^{m}=\partial \xi^{m} / \partial x^{\mu}$ and $e_{l}^{\lambda}=\partial x^{\lambda} /\left.\partial \xi^{l}\right|_{\xi=\xi(x)}$ are the frames relating the distinguished globally affine coor dinates and the arbitrary observer's ones in terms of the $d$ nondynamical generally covariant scalar fields $\xi^{m}(x)$ (having the inverse $x^{\mu}(\xi)$ ). This is the least set of free functions to consistently account for the GR violation, under preserving general covariance. The topology of the affine background, flat vs. curved, is thus not a pure theoretical question but becomes, in principle, liable to observational verification ${ }^{15}$. Anyhow, the flat affine background determined by Equation (25) could be treated, in a space-time region, as an approximation to a curved affine background determined by a regular $\hat{\Gamma}_{\mu \nu}^{\lambda}{ }^{16}$.

\subsection{Unimodular Relativity}

For $\Delta L_{1}$ one has

$$
B_{\mu \lambda}^{\lambda}=\partial_{\mu} \ln \sqrt{|g|}-\hat{\gamma}_{\mu},
$$

where $\hat{\gamma}_{\mu} \equiv \hat{\Gamma}_{\mu \lambda}^{\lambda}$. Moreover, if the affine background is flat, one gets in view of Equation (25):

$$
\hat{\gamma}_{\mu}=e_{l}^{\lambda} \partial_{\mu} e_{\lambda}^{l}=\partial_{\mu} \ln \left|\operatorname{det}\left(e_{\lambda}^{l}\right)\right| .
$$

Thus under this limitation one has

$$
\Delta L_{1}=g^{\mu v} \partial_{\mu} \varsigma \partial_{\nu} \varsigma
$$

where

$$
\varsigma=\ln (\sqrt{|g|} / \hat{\mu})
$$

with

$$
\hat{\mu}=\left|\operatorname{det}\left(\partial_{\mu} \xi^{m}\right)\right| .
$$

The field $\varsigma$, determined by the ratio of the two scalar densities of the same weight, behaves like a generally covariant scalar. The nondynamical field $\hat{\mu}$ being a unimodular scalar, the theory with only $\Delta L_{1}$ (in addition to $L_{0}$ ) may for uniformity be referred to as Unimodular Relativity (UR), with the scalar graviton $\varsigma$ serving as

\footnotetext{
${ }^{15}$ Under restriction $a b$ initio by GR, the type of the affine background, flat or curved, would superficially seem irrelevant.

${ }^{16} \mathrm{~A}$ limiting generally noncovariant case corresponding here, in this paper, to GR violation with $\hat{\Gamma}_{\mu \nu}^{\lambda}=0, \quad \xi^{m}=\delta_{\mu}^{m} x^{\mu}$ and $e_{\mu}^{m}=\delta_{\mu}^{m} \quad$ was elaborated, irrespective of DM, in [13].
} 
DM [9] [10]. The scalar graviton proves now to be nothing but a Goldstone boson corresponding to the hidden dilatation symmetry ${ }^{17}$. Besides, the so-called "modulus" $\hat{\mu}$ acquires the clear-cut physical meaning ${ }^{18,19}$. To qualitatively compile with the astrophysical data on the galaxy anomalous rotation curves due to the dark halos, there should fulfill $\varepsilon_{1}^{1 / 2} \sim v_{\infty} \sim 10^{-3}$, with $v_{\infty}$ being an asymptotic rotation velocity. To tame phenomenologically the possible unwanted properties of the gravitational DM, a hierarchy of the GR violations $\left|\varepsilon_{2-5}\right| \ll\left|\varepsilon_{1}\right| \ll 1$ may be envisaged, with GR substituted first by UR and ultimately by VR.

\section{Conclusion}

The nonlinear affine Goldstone model may provide a prototype for the emergent gravity, with the GR violation and the gravitational DM serving as the signatures of emergence. The proper account for a nondynamical affine connection, missing in GR, proves to be crucial. The main outcome of the model is the effective Lagrangian for the emergent metric gravity given by Equations (17), (21) and (22) (supplemented in the limited version by Equation (25)). Resulting in VR, the model widely extends the phenomenological horizons beyond GR, with the possible substitution of GR first by UR and then by VR. In an ultimate theoretical perspective, the model may, hopefully, serve as a guide towards a putative underlying theory of gravity and space-time.

\section{Appendices}

\subsection{Spontaneous Symmetry Breaking and Nonlinear Realizations}

Let us shortly recapitulate the techniques of the nonlinearly realized/hidden symmetries. Let a global continuous internal symmetry $G$, with the dimension $d_{G}$, be spontaneously/dynamically broken, $G \rightarrow H$, to some $d_{H}$-dimensional subgroup $H \subset G$. Let $K=G / H \subset G$ be the respective $d_{K}$-dimensional (for definiteness, left) coset space consisting of the (left) coset elements $k \in K$. Then any group element $g \in G$ admits a unique (at least in a vicinity of unity) decomposition $g=k h$, with $k \in K$ and $h \in H$. Henceforth under the action of a group element $g_{0}$, one should get $g^{\prime}=g_{0} k=k^{\prime} h^{\prime}\left(g_{0}, k\right)$. The group $G$ thus acts on $k$ by means of the transformations $k \rightarrow k^{\prime}=g_{0} k h^{\prime-1}\left(g_{0}, k\right)$ dependent, generally, on $k$ (henceforth the term nonlinear). Mapping a flat space $R^{d}$ onto $K, R^{d} \rightarrow K$, defines on $R^{d}$ a coset-valued field $k(\xi) \in K, \xi \in R^{d}$. This induces a nonlinear realization of $G$ on $K$. Restricted by the unbroken subgroup $H$, i.e., under $g_{0}=h_{0}$, the nonlinear realization of $G$ is to be a usual linear representation of $H, k \rightarrow k^{\prime}=h_{0} k h_{0}^{-1}$, with $h^{\prime}\left(h_{0}, k\right)=h_{0}$ and $h^{\prime}(I, k)=I$. Putting $k=\exp \left(\sum_{i} \pi_{i} X_{i}\right), \quad i=1, \cdots, d_{K}, d_{K}=d_{G}-d_{H}$, with $X_{i}$ being the broken generators of $G$, one can treat the $d_{K}$-component field $\pi$ as a Goldstone boson emerging under the global symmetry breaking. Due to the isomorphism $G \simeq K \otimes H$ (at least in a vicinity of unity), one can substitute a coset element $k$ by its equivalence class $\kappa$ obtained from $k$ through the (right) multiplication by an arbitrary $h \in H$. At that, the nonlinear realization gets linearized as $\kappa \rightarrow \kappa^{\prime}=g_{0} \kappa h^{-1}$, modulo an arbitrary $h(\xi) \in H_{\text {loc }}$ independent of $\kappa$. And v.v., fixing a gauge for $H_{\text {loc }}$ results in imposing the $d_{H}=d_{G}-d_{K}$ restrictions on $\kappa$ and choosing thus a nonlinear realization $k \rightarrow k^{\prime}=g_{0} k h^{\prime-1}\left(g_{0}, k\right)$. Hence, being equivalent to the linear representation, all the nonlinear realizations for breaking $G \rightarrow H$ are equivalent among themselves in the effective field theory sense. At that, the linearization of a hidden symmetry may be more advantageous as embodying on par all the equivalent nonlinear realizations.

\subsection{Conventions and Notations}

For convenience, let us present a short compendium of conventions and notations used in the paper. For generality, the dimension of space-time is assumed $d \geq 2$, with the temporal-spacial signature $(1, d-1) . \mathcal{R}_{d} \simeq R^{d}$ is a representation space/a flat affine background covered with the globally affine coordinates $\xi^{m}$. The indices $m, n, \cdots=0,1, \cdots, d-1$ undergo the affine transformations through $A_{m}^{n} \in G L(d, R)$.

$\vartheta_{m}^{a}(\xi) \in G L(d, R) / S O(1, d-1)$ is a coset field on $\mathcal{R}_{d}$. The indices $a, b, \cdots$ undergo the global Lorentz

\footnotetext{
${ }^{17}$ Such a specific dilaton in disguise, representing a compression gravity mode in metric, may be called the "systolon” [10].

${ }^{18}$ It proves that the unknown modulus $\hat{\mu}$ may be hidden into $\varsigma$ taken as an independent field variable and, after finding the latter together with metric through the field equations, be reconstructed as a consistency condition. The proper solutions may be associated with the scalar-graviton DM. The generally covariant formalism is crucial to this end [9] [10].

${ }^{19} \mathrm{~A}$ theory of gravity (the so-called "TDiff gravity") in the generally noncovariant form corresponding here, in this paper, to UR in the gauge $\hat{\mu}=1$ was elaborated, irrespective of DM, in [14].
} 
transformations $\Lambda_{a}^{b} \in S O(1, d-1) \subset G L(d, R) . \mathcal{M}_{d}$ is a curved world manifold covered with the observer's coordinates $x^{\mu} . \mu, v, \cdots$ are the world indices undergoing the diffeomorphism transformations through $J_{v}{ }^{\mu}=\partial x^{\mu} / \partial x^{\prime \nu}$ produced by the general coordinate changes $x^{\mu}=x^{\mu}\left(x^{\prime}\right) . \hat{\Gamma}_{\mu v}^{\lambda}$ is a nondynamical background affine connection on $\mathcal{M}_{d}$. $\xi_{X}^{m}$ are the distinguished locally affine coordinates on $\mathcal{M}_{d}$, attached to an arbitrary point $X$, with $\left.\hat{\Gamma}_{m n}^{l}\right|_{X}=0 . e_{\mu}^{m}=\partial \xi_{X}^{m} / \partial x^{\mu}$ is the respective locally affine frame. $\Gamma_{\mu \nu}^{\lambda}$ is the dynamical Christoffel connection on $\mathcal{M}_{d}$, corresponding to the emergent metric $g_{\mu v}$. The metric is constructed from a pair of the locally Lorentzian frames $\vartheta_{\mu}^{\alpha}(x)$ treated as the true gravity field on $\mathcal{M}_{d}$. The indices $\alpha, \beta, \cdots$ undergo the local Lorentz transformations $\Lambda_{\alpha}^{\beta}(x) \in S O(1, d-1)_{\mathrm{loc}}$.

\section{References}

[1] Sindoni, L. (2012) SIGMA, 8, 27-71 [arXiv:1110.0686 [gr-qc]]

[2] Carlip, S. (2012) Challenges for Emergent Gravity. arXiv:1207.2504 [gr-qc]

[3] Coleman, S.R., Wess, J. and Zumino, B. (1969) Physical Review D, 177, 2239-2250.

[4] Salam, A. and Strathdee, J. (1969) Physical Review D, 184, 1750-1759.

[5] Isham, C., Salam, A. and Strathdee, J. (1971) Annals of Physics (New York), 62, 98-119. http://dx.doi.org/10.1016/0003-4916(71)90269-7

[6] Borisov, A.B. and Ogievetsky, V.I. (1974) Teoreticheskaya i Matematicheskaya Fizika (in Russian), 21, 329-342. http://dx.doi.org/10.1007/BF01038096

[7] Sardanishvily, G. (1980) Physics Letters A, 75, 257-258. http://dx.doi.org/10.1016/0375-9601(80)90555-1

[8] Pirogov, Yu.F. (2005) Physics of Atomic Nuclei, 68, 1904-1915 [Yad. Fiz., 68, 1966-1977] [arXiv:gr-qc/0405110] http://dx.doi.org/10.1134/1.2131118

[9] Pirogov, Yu.F. (2005) Physics of Atomic Nuclei., 69, 1338-1344 [Yad. Fiz., 69, 1374-1380] [arXiv:gr-qc/0505031] http://dx.doi.org/10.1134/S1063778806080102

[10] Pirogov, Yu.F. (2012) The European Physical Journal C, 72, 2017-2039 [arXiv:1111.1437 [gr-qc]]

[11] Anderson, J.L. (1971) General Relativity and Gravitation, 2, 161-172. http://dx.doi.org/10.1007/BF02450447

[12] Van Dam, H. and Ng, Y.J. (2001) Physics Letters B, 520, 159-162 [arXiv:hep-th/0108067] http://dx.doi.org/10.1016/S0370-2693(01)01140-6

[13] Anber, M.M., Aydemir, U. and Donoghue, J.F. (2010) Physical Review D, 81, 084059 [arXiv:0911.4123 [gr-qc]]

[14] Alvarez, E., Blas, D., Garriga, J. and Verdaguer, E. (2006) Nuclear Physics B, 756, 148-170 [arXiv:hep-th/0606019] http://dx.doi.org/10.1016/j.nuclphysb.2006.08.003 
Scientific Research Publishing (SCIRP) is one of the largest Open Access journal publishers. It is currently publishing more than 200 open access, online, peer-reviewed journals covering a wide range of academic disciplines. SCIRP serves the worldwide academic communities and contributes to the progress and application of science with its publication.

Other selected journals from SCIRP are listed as below. Submit your manuscript to us via either submit@scirp.org or Online Submission Portal.
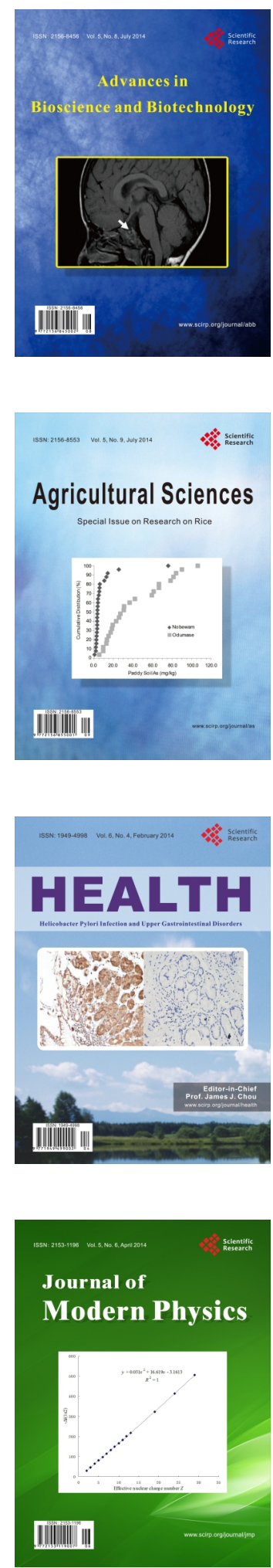
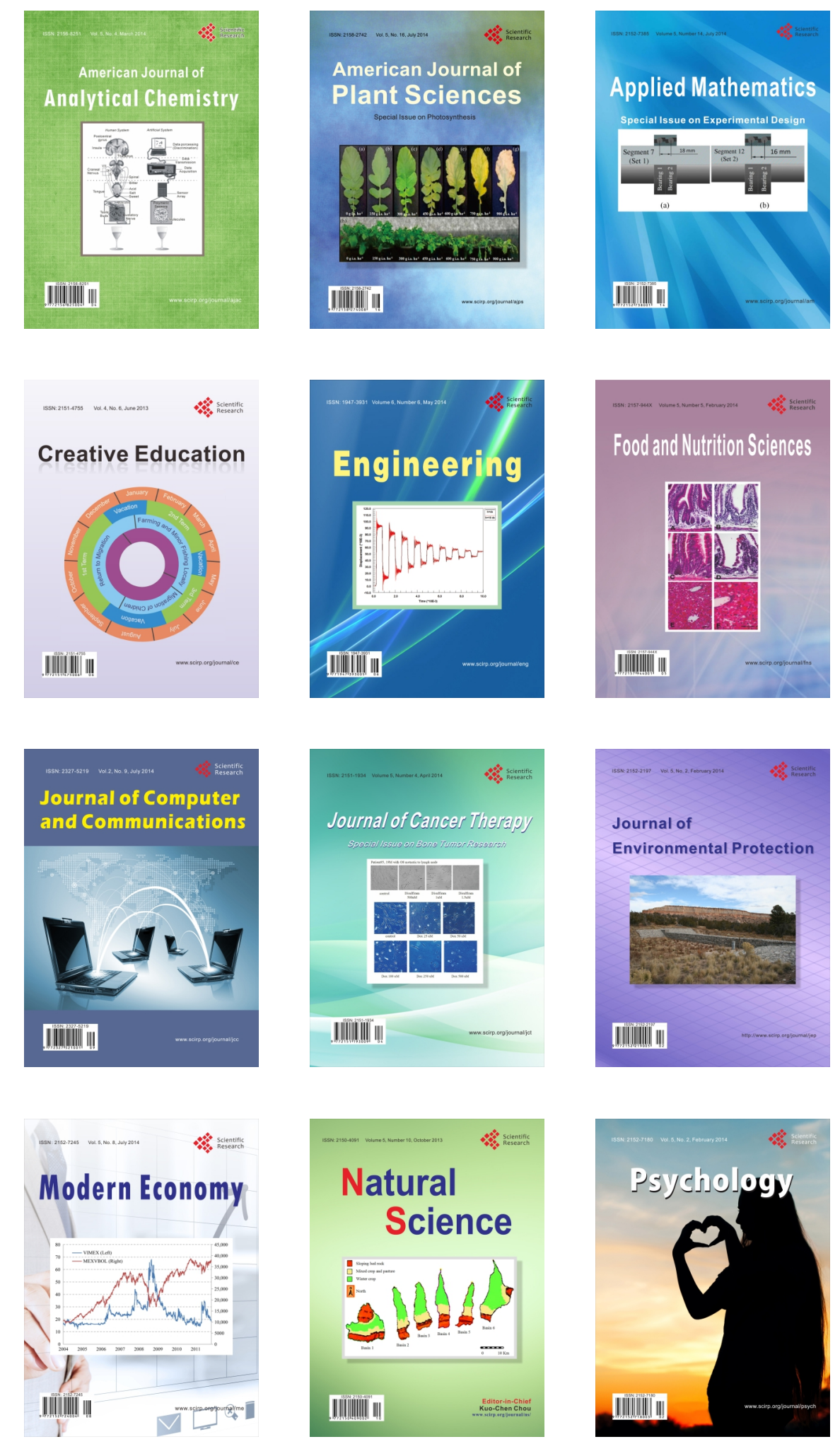